\title{
Enhancements of the Flow Solver TAU for Internal Flows at Supercritical Pressures
}

\author{
Rudolph, Martin \\ German Aerospace Center (DLR) \\ Institute of Aerodynamics and Flow Technology - Section Spacecraft \\ Lilienthalplatz 7, 38108 Braunschweig, GERMANY \\ martin.rudolph@dlr.de
}

\begin{abstract}
This present paper discusses numerical investigations to verify the real fluid solver version of the DLR Navier-Stokes code TAU. Longterm goal is the simulation of real fluid cooling channel flows for liquid rocket engines (LREs). To verify the infrastructure of the code, a numerical micro-tube flow test case based on methane is selected. Due to severe problems in solving the stiff thermodynamics for real propellants and that security restrictions for experiments impede the use of hydrogen as propellant, most of the validation experiments are conducted with nitrogen. The use of nitrogen under supercritical pressure is an accepted strategy towards high pressure liquid rocket engine flows. To enable the computations, a modified Benedict-Webb-Rubin (MBWR) real fluid thermodynamic treatment and several boundary conditions have been implemented accounting for high pressure internal flow with heat transfer. Fluid properties have been chosen to supercritical pressure and subcritical temperatures at the inflow and supercritical pressure and temperature at the outflow due to heating. This ensures fluid conditions in the pseudo-critical region. In conclusion it can be shown that the TAU code is suitable to deal with real fluid convective heat transfer, although there are still a few stability concerns regarding realistic propellants like hydrogen and methane. By changing the infrastructure of the MBWR lookup table it is, eventually, possible to get a good trade-off between stability and performance.
\end{abstract}

Keywords: Supercritical fluid, Pseudo-critical, Turbulent pipe flow, Heat transfer

\section{Introduction}

The design of liquid rocket engine (LRE) subsystems which are subjected to high thermal loads is a challenging task, even nowadays. Due to the extremely large combustion enthalpies of modern liquid propellants, the combustion chamber walls are subjected to extreme heat fluxes. To keep the walls well below their limits, active cooling is necessary. In recent LREs, regenerative cooling is the most popular technology. The design of an efficient cooling process needs a huge variety of costly experiments. For the sake of risk and cost reduction, numerical simulations of engine subsystem are mandatory.

Within the frame of the DLR project ProTAU, the in-house code TAU shall be improved to simulate all subsystems of LREs, e.g. combustion chamber, nozzle and cooling channels. One goal of the project is the numerical investigation of real propellant high aspect ratio cooling channel flows by means of multidisciplinary coupling of CFD and a finite element method for the thermal field. Enabling the CFD code for internal real fluid flows is one of the most challenging tasks, because the TAU code is mainly designed for external flows. A variety of new boundary conditions for internal flows is needed and fluid properties have to be properly modelled.

Simulating cooling channel flows with real propellants like hydrogen reveals stability issues due to the stiff thermodynamics. In almost the same manner, performing experiments with real propellants is challenging. Verifying fluid property modelling and solver boundaries using nitrogen is an accepted way. On the experimental side, nitrogen is very easy to handle and due to relatively high critical temperatures, it is fairly simple to span a huge range of fluid properties. On the numerical side, nitrogen real fluid thermodynamics are better-natured than real propellants. Verification of solver infrastructure is easier using nitrogen.

\section{The DLR TAU Code}

All of the present simulations have been carried out using the DLR in house code TAU, which is a hybrid grid, Godunov-type finite volume CFD code. It solves the integral form of the Navier-Stokes or Euler equations. TAU is of second order accuracy in space. The Department Spacecraft is responsible for the development of addons for spacecraft applications, including thermochemical equilibrium and non-equilibrium. TAU has been validated for a variety of steady and unsteady flows in sub-, trans-, super- and hypersonic Mach number regimes, see Mack \& Hannemann [1] and Gerhold et al. [2].

Based on the ideas of supercritical fluid behaviour [3], Banuti developed a two-phase real fluid model which is capable of calculating phase changes and mixtures of a real fluid with ideal gases [4]. This real-gas extensions consist mainly of two parts: A separate preprocessing addon, which generates a database with fluid properties over $\rho-T$ and a thermodynamic library which solves the dominating $\rho-e$-problem and some auxiliary thermodynamic problems like $\rho-p$ and $p-T$.

\section{Numerical setup}

In the abscence of experimental data, a literature research revealed, that there are only few experimental setups which provide sufficient data and boundary conditions. Wang et al. investigated the turbulent convective heat transfer numerically for methane, see [5]. As a verification and validation step, a methane microtube flow with exactly the same numerical domain as seen in Figure 1 was simulated. Unfortunately, our solver reveals massive stability issues for cryogenic methane at supercritical pressures, which mainly is a 
result of the extremely stiff thermodynamics. Therefore, as verification of the real fluid solver TAU, a nitrogen flow through the same pipe configuration as found in Wang et al. [5] is considered. The fluid property regime is set to comparable properties with regard to the critical point, see Table 1. Regarding the same regions $\left(P / P_{c}, T / T_{c}\right)$ in the phase diagram leads to comparable heat transfer phenomena.

Table 1: Critical fluid properties for methane and nitrogen.

\begin{tabular}{r|cc|cc|cc|}
\hline Fluid & $P_{c}[\mathrm{bar}]$ & $T_{c}[\mathrm{~K}]$ & $P[\mathrm{bar}]$ & $T[\mathrm{~K}]$ & $P / P_{c}$ & $T / T_{c}$ \\
\hline Nitrogen & 33.96 & 126.26 & 40.00 & 100.00 & 1.178 & 0.792 \\
Methane & 45.99 & 190.56 & 60.00 & 140.00 & 1.305 & 0.735 \\
\hline
\end{tabular}

\subsection{Geometry and Boundaries}

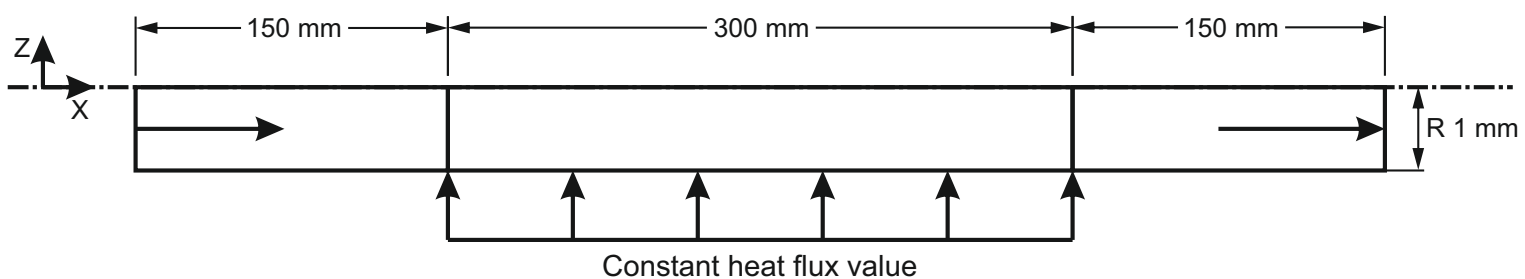

Fig. 1: Computational Domain for the investigations.

According to [5], the computational domain is a 2D axisymmetric grid of a $2 \mathrm{~mm}$ diameter microtube. The overall length of the domain is $600 \mathrm{~mm}$ with a $300 \mathrm{~mm}$ heated section in the middle (Fig. 1). The boundaries are viscous walls on the bottom surface, a symmetry axis at the top and symmetry planes on the sides (in-plane in Figure 1). On the heated section of the pipe outer wall, several heat flux values of $\dot{q}=[1,3,5,7] \mathrm{MW} / \mathrm{m}^{2}$ are applied. The inflow boundary is assumed as massflux boundary, where the massflux $\rho u$ and the static temperature $T_{\text {stat }}$ are specified. The pressure $p$ is extrapolated from the fluid domain. That leads to a separate treatment of the thermodynamic $p$-T-problem. The inflow massflux is set to a resulting inflow axial velocity of $u \approx 100 \mathrm{~m} / \mathrm{s}$, the static temperature is adjusted to $T_{\text {stat }}=100 \mathrm{~K}$, leading to a Mach number of $M=0.12$. Smaller intended Mach numbers take long computational time, because preconditioning is not supported by the real fluid solver TAU. The outflow is set as exit pressure boundary, with a pressure value of $p=40$ bar.

The resulting 2D axisymmetric grid consists of 606202 grid points $(3001 \times 101 \times 2$ grid points in axial, radial and circumferential direction, respectively). The first grid spacing at the viscous wall aims for $y^{+}<1$ at the cold wall, leading to a $y^{+}<0.08$ at the heated wall. The grid resolution is sufficient to resolve all existing gradients.

\subsection{Nitrogen Library}

As mentioned before, the equation of state (EOS) is stored in a NetCDF lookup table as a preprocessing step. The solver allows various EOS, i.e. Peng-Robinson (PR), European Gas Research Group (GERG) and Modified Benedict-Webb-Rubin (MBWR). For the present investigations the MBWR EOS is chosen because of its accuracy compared to the experimental database of ${ }^{1}$ NIST, see [4] and [3]. Detailed information about the equation of state can be found in [6].

The fluid property database is represented by a structured grid over density and temperature, both being distributed logarithmically. That results in an extremely fine grid for small densities and temperatures. This stems from the fact, that it has been designed for supercritical injection in the combustion chamber. Accounting for chemical reactions means, that partial densities of the species tend to zero. To avoid having huge equidistant lookup tables, a logarithmic distribution is applied. In the present study, the inflow is subjected to low temperature (subcritical, $T=0.792 T_{c}$ ) and high pressure (supercritical, $P \geq 4 P_{c}$ ), leading to high densities. The resulting nitrogen database spans a temperature range of $T=[80 \cdots 600] \mathrm{K}$, the density spans a range of $\rho=[1 \cdots 820] \mathrm{kg} / \mathrm{m}^{3}$ with $800 \times 800$ grid points in density and temperature, respectively. The critical point is sufficiently resolved (Fig. 2). The database generation prior to the calculation takes only a few minutes, but saves a lot of computational time during calculation.

\section{Results}

As mentioned before, the present investigations are performed at an outlet pressure of 40 bar and an inlet temperature of $100 \mathrm{~K}$. The fluid mass flux corresponds to a axial velocity of $u=100 \mathrm{~m} / \mathrm{s}$. The resulting inflow Mach number is about $M=0.12$. The first calculation has been conducted using a heat flux value of $\dot{q}=1 \mathrm{MW} / \mathrm{m}^{2}$, followed by a stepwise increase in heat flux up to $\dot{q}=7 \mathrm{MW} / \mathrm{m}^{2}$. The outlet pressure and inflow temperature are constant for all cases. Figure 3 qualitatively shows the temperature distribution for the high heat flux condition. A notable increase in near wall fluid temperature is observed. The reddish area at the end of the heated section $(X=0.3 \mathrm{~m}, X / D=150)$ needs further investigation. The ratio between radial and axial direction is exaggerated, but the thermal boundary layer is not linear anymore.

Figure 4 depicts the pressure-temperature trajectory, starting at high pressures and low temperatures. By passing through the adiabatic part of the pipe only pressure loss is observed, the wall temperature stays roughly constant. Passing the constant heat flux

${ }^{1}$ National Institute of Standards and Technology, http://http://webbook.nist.gov/chemistry/fluid/ 


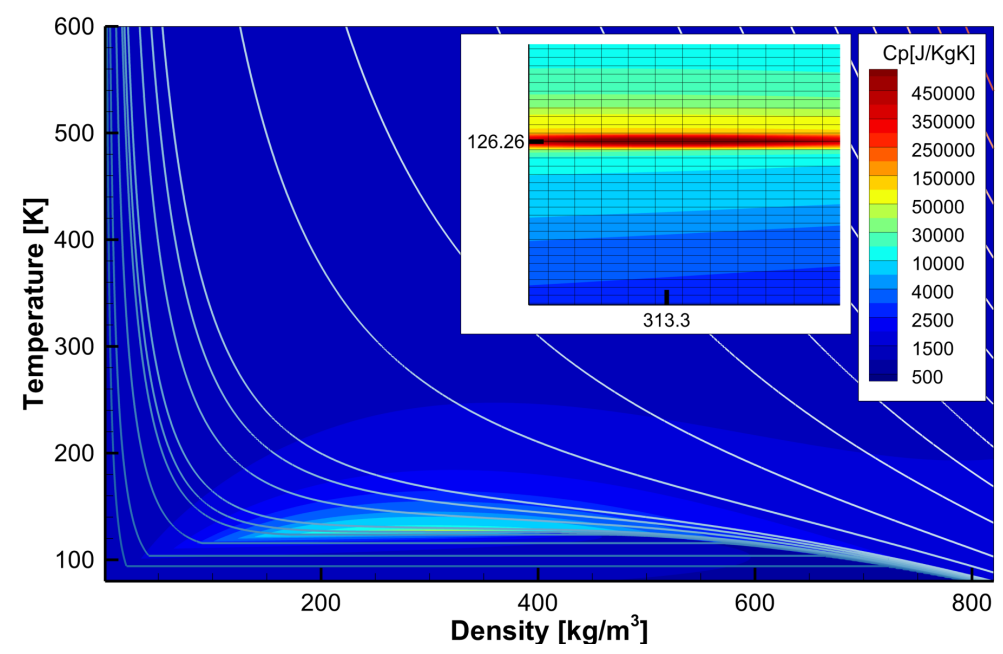

Fig. 2: MBWR contours of isobaric heat capacity in the density-temperature primary fluid property domain with pressure-coloured isobaric lines, details around the critical point with grid depicted in secondary frame.

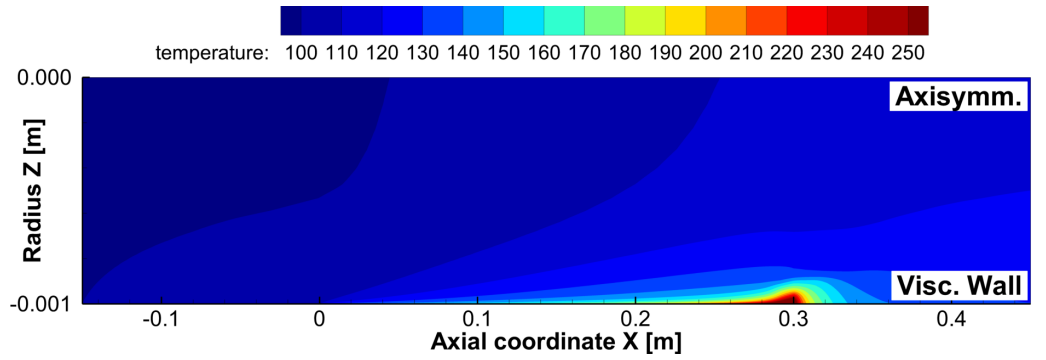

Fig. 3: Temperature distribution in pipe symmetry plane for $q=7 \mathrm{MW} / \mathrm{m}^{2}$.

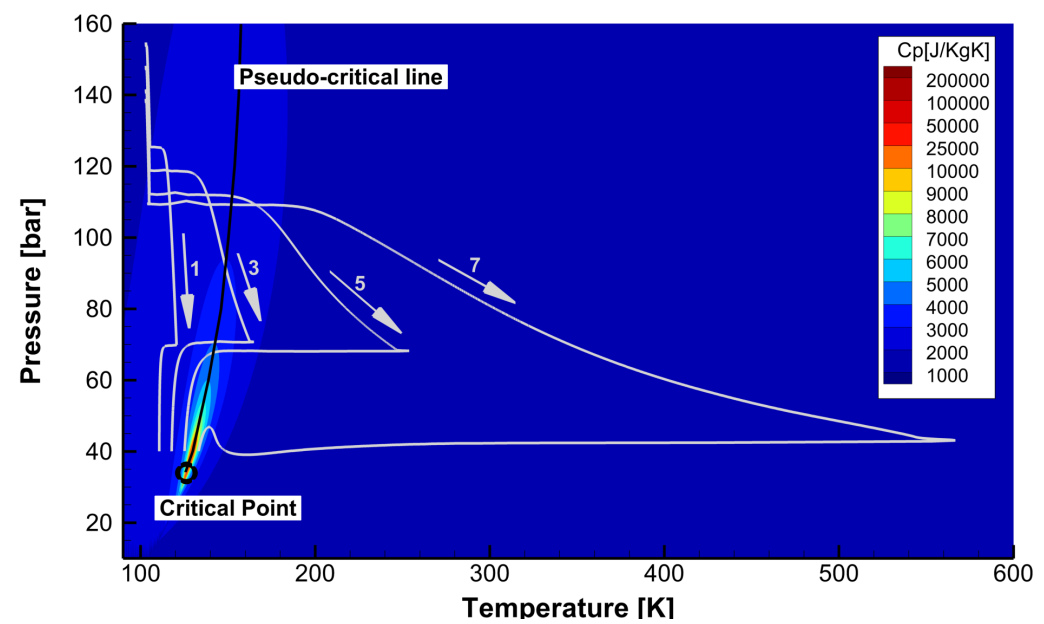

Fig. 4: MBWR contours of isobaric heat capacity in the temperature-pressure fluid property domain, grey lines indicate the pressure and wall temperature for heat flux values between $1 \mathrm{MW} / \mathrm{m}^{2}$ and $7 \mathrm{MW} / \mathrm{m}^{2}$.

viscous wall, the temperature increases depending on the heat flux value. The pressure is decreasing further. The trajectory trends towards perfect gas behaviour. The pipe tail end again has an adiabatic viscous wall. The wall temperature drops towards adiabatic wall 
temperature, being slightly different for the different heat flux values. The heated section causes an increase in bulk temperature, leading to different wall temperatures. Only for the $7 \mathrm{MW} / \mathrm{m}^{2}$ case, there is a sudden pressure increase at the end of the pipe, which does not seem very physical and needs further investigation.

Better insight into the physics can be gained by regarding Figure 5, which provides detailed plots of main fluid and flow properties. Figure 5a shows the pressure and Mach number distribution along the symmetry axis of the pipe. Outflow pressure and inflow Mach number are exactly the same for all heat flux values. But the four different heating scenarios result in diverging pressure loss over the heated section. In the adiabatic inflow section of the pipe, pressure losses are identical for all cases. The Mach number in contrast shows

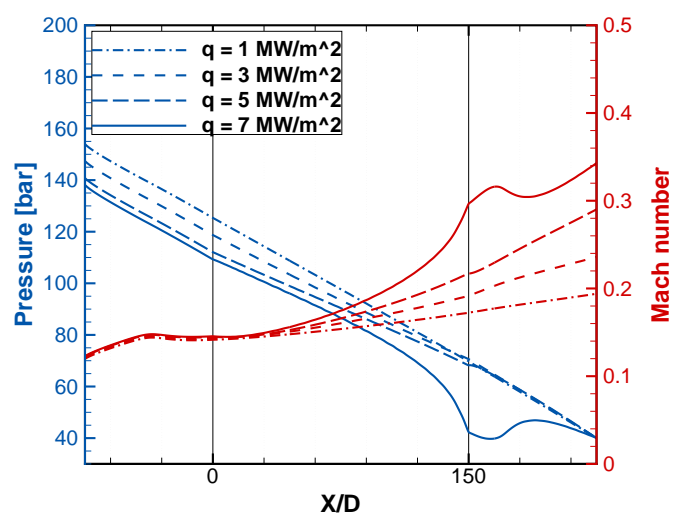

(a) Pressure (left) and Mach number (right yaxis)

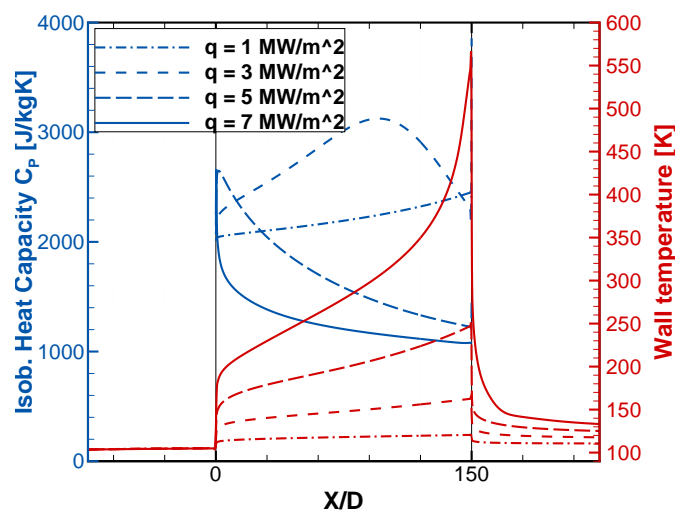

(c) Isob. Heat Capacity on the heated wall (left) and wall temperature (right y-axis)

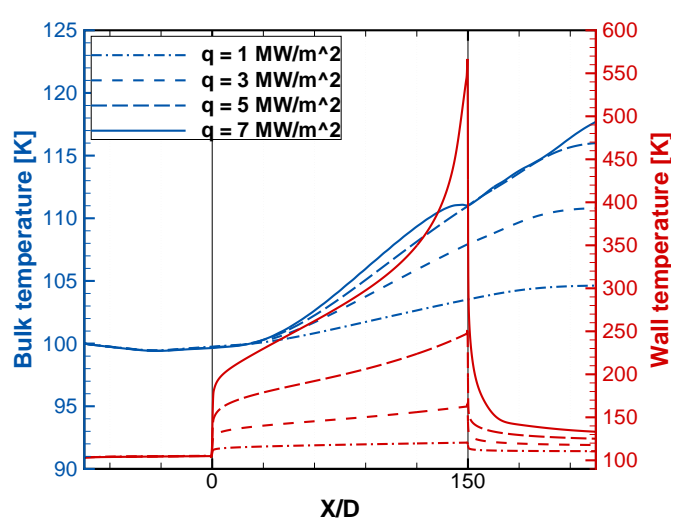

(b) Bulk (left) and Wall temperature (right yaxis)

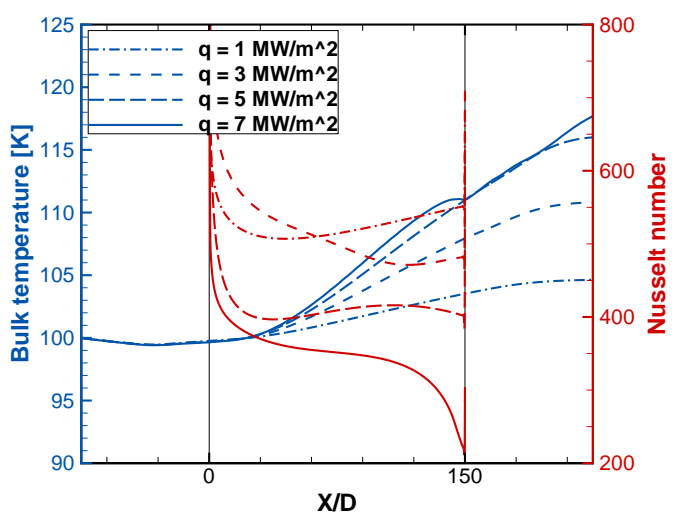

(d) Bulk temperature (left) and Nusselt number on the heated wall (right y-axis)

Fig. 5: Fluid properties over dimensionless pipe length for different heat flux values on the heated section.

similiar behaviour in the adiabatic inflow section, the heating causes a different axial gradient of the Mach number. Due to different bulk temperatures in the adiabatic tail section and different resulting speeds of sound, the axial Mach gradient is not identical for the four cases. For the high heat flux case, the aforementioned pressure increase in the tail section is observable again. Figure 5b shows bulk and wall temperature over the dimensionless axial direction $X / D$. The bulk temperatures diverge only slightly for the four heat flux scenarios. The wall temperature, in turn, increases massively for high heat fluxes and nearly reaches the temperature bounds of the fluids MBWR lookup table, used in that case. However, the $600 \mathrm{~K}$ are within the validity range given by NIST.

To further investigate the possible anomalies, Figures $5 \mathrm{c}$ and $5 \mathrm{~d}$ can be consulted. Fig. $5 \mathrm{c}$ shows the isobaric heat capacity $C_{P}$ at wall temperature $T_{w}$. The extremely low isobaric heat capacity of the fluid for high heat flux condition leads to a large temperature increase. Dimensional analysis reveals a strong increase in wall temperature for nearly constant mass and constant energy being transferred. Fig. 5d shows bulk temperature and Nusselt number over the dimensionless axial coordinate $X / D$. The Nusselt number minimizes at the end of the heated section for high heat fluxes. The small Nusselt number is equivalent to a small temperature gradient away from the wall. This is a reasonable explanation for the significant high-temperature area (reddish area in Fig. 3). This, again, leads to a decrease in speed of sound and increase in Mach number. The local Mach number in the high-temperature region reaches $M=0.9$. As a result, the pressure distribution for the high heat flux case massively differs from lower heat flux cases.

\section{Conclusion and Outlook}

This paper reports on a first numerical verification step for the TAU code towards predicting cooling channel flows in realistic liquid rocket engines. A simple nitrogen pipe flow test case subjected to a constant wall heat flux is investigated. The results show, that 
the TAU code is capable of calculating real fluids. Further investigations to verify and validate the code with regard to real propellants like hydrogen are under way. Furthermore, a proper roughness modelling for the thermal boundary layer of realistic cooling channel configurations has to be introduced in TAU. For stabilization and runtime optimization purpose, the infrastructure of the real fluid lookup table should be changed to better represent the perfect gas behaviour for high temperatures. A better performance of the code for extremely low Mach numbers could be gained by implementing an adequate preconditioning algorithm for real fluids.

\section{References}

[1] A. Mack and V. Hannemann, "Validation of the Unstructured DLR TAU-Code for Hypersonic Flows," in 32nd AIAA Fluid Dynamics Conference and Exhibit, St. Louis, MO, 2002.

[2] T. Gerhold, M. Galle, O. Friedrich, and J. Evans, "Calculation of complex three-dimensional configurations employing the DLRTAU-code," in Proceedings of the 35th Aerospace Sciences Meeting and Exhibit, Reno, NV, 1997.

[3] J. B. Calvo and K. Hannemann, "Numerical simulation of liquid rocket engine cooling channels," in Proceedings of the 45th AIAA/ASME/SAE/ASEE Joint Propulsion Conference \& Exhibit, Denver, CO, 2009.

[4] D. Banuti, "Thermodynamic Analysis and Numerical Modeling of Supercritical Injection," Ph.D. dissertation, Inst. Aerospace Thermodynamics, Univ. of Stuttgart, Germany.

[5] Y.-Z. Wang, Y.-X. Hua, and H. Meng, "Numerical Studies of Supercritical Turbulent Convective Heat Transfer of CryogenicPropellant Methane," J. Thermophys Heat Tr., vol. 24, no. 3, pp. 490-500, 2010.

[6] B. A. Younglove, "Thermophysical Properties of Fluidsi̇̇̇rgon Ethylene Parahydrogen Nitrogen Nitrogen Trifluoride and Oxygen," J. Phys. Chem. Ref. Data, vol. 14, no. 2, pp. 619, 1985. 